\title{
Family variables and child mental disorders in a Third World urban area (Bahia, Brazil)*
}

\author{
Naomar de Almeida-Filho \\ Department of Preventive Medicine, Faculty of Medicine, University of Bahia, Brazil \\ Researcher (II-C) of the National Council for Scientific and Technological Development (CNPq), Brazil
}

\begin{abstract}
Summary. The relationship between family support variables and child mental disorders was assessed through a prevalence study conducted in an urban neighborhood of Bahia, Brazil. From a representative sample of 828 children, aged between 5 and 14 years, $23.3 \%$ were diagnosed as having varying degrees of psychological symptoms. Diagnoses were based on psychiatric interviews, following screening using the Questionário de Morbidade Psiquiátrica Infantil (QMPI). Results supported the hypotheses that: (a) children from smaller families exhibit a higher prevalence than those from larger families; (b) prevalence of neurotic and psychosomatic disorders is lower for children from larger families than for those from smaller ones. The data failed to confirm the hypothesis that family type is associated with the occurrence of psychopathology in children. Controlled analyses indicated that these relationships were not confounded by the age or sex of the child, the social class, type or size of the family.
\end{abstract}

The social support hypothesis has emphasized the role of the family as a primary source of protective factors related to the onset of mental disorders in general (Kaplan et al. 1977), declaring that the availability and quality of emotional and material supports affects susceptibility to the negative health consequences of stress. Social support can be viewed as a mediator between the individual and a stressful environment, possibly exerting a protective effect against stress-related mental disability. As the family is a major source of social support, despite crowded

\footnotetext{
* This research was supported by the Masters Program in Community Health of the University of Bahia and by the Rockefeller Foundation.
}

living conditions in many large families, the availability of its members could be related to better child mental health.

The social support hypothesis thus lends credence to the role of family type as a protective or "risk" factor relevant to mental disorders in general, but formulations have not been extended to examine its effect on children's mental health. Research conducted in developed societies has explored mental disorders in children from the perspective of maternal deprivation (Rutter 1972), birth oder (Touliatos and Lindholm 1980), child spacing (Hendricks 1977), and family relationships (Nye 1958). Little attention, however, has been paid to the potentially protective character of the extended family, since it is not a predominant cultural feature in these countries (Bossard and Boll 1956).

On the other hand, the association between family size and child mental health has been examined by several investigators. Studies have been reported, which have found negative relationships, no relationship, and positive relationships between family size and child mental health. Children and adolescents from larger families have been shown to be less well adjusted than those in smaller families (Hawkes et al. 1958; Nuttall et al. 1971; Nye 1952, 1958). Tuckman and Regan (1967) found increasing levels of antisocial behavior in children as family size increased. High levels of stress in children from large families have also been found by Leighton (1972) and Suepsaman (1973).

Findings of poorer child adjustment in large, extended families could reflect such factors as decreased quantity and quality of maternal care (Spence et al. 1954), or increased complication and disorganization of family relationships and roles (Christensen 1968). Members of large, extended families may also experience stress related to over- 
crowded living conditions (Hendricks 1977). Crowding has been linked with increased stress (Hassan 1977), juvenile delinquency (Schmitt 1966), and child antisocial behavior (Chombart de Lauwe 1960). Children lacking the ability to significantly alter their environment could be most susceptible to the negative effects of crowded living conditions (Baldassare 1979; Hassan 1977). Cassel (1971, 1973, 1976), however, after reviewing a number of studies on crowding, concluded that no relationship, or an inverse relationship, should exist between crowding and mental health. In support of this conclusion, no relationship between family size and child mental health was found by Hendricks (1977) or by Touliatos and Lindholm (1980). Children from large, extended families have also been shown to exhibit lower rates of emotional disturbance (Ellis and Beechley 1951), more favorable personality development (Bossard and Boll 1956), and better social adjustment than children from small families (Landis 1954).

Some of the reported investigations have used small samples (Bossard and Boll 1956; Hawkes et al. 1958; Hendricks 1977), only clinic populations (Ellis and Beechley 1951; Tuckman and Regan 1967), or convenience samples (Nye 1958; Templeton 1962). Further, some researchers have not adequately described sampling methods (Landis 1954; Nuttall et al. 1971; Touliatos and Lindholm 1980), or definitions of outcome variables (Hawkes et al. 1958; Ellis and Beechley 1951; Bossard and Boll 1956). None of these studies employed methodological procedures adequate for the assessment of risk of child mental disorders from an epidemiological standpoint, and few have considered the effect of extraneous factors in their analyses.

As a whole, these investigations were descriptive rather than utilizing explicit theoretical frames of reference. Exceptions were studies by Hendricks (1977), who integrated his hypothesis into a stress/ density/crowding framework, Nuttall et al. (1971), who interpreted their results in terms of intrafamily dynamics, and Suepsaman (1973), who claimed a broader life crisis stress theory to explain her findings.

The present study examined the relationships between family type, family size and child mental disorders (CMD) in a sample of children living in a lowincome urban area of a Third World city. Based on the findings of previous research and inspired by the social support hypothesis, larger, extended families were expected to be associated with better child mental health status. The following hypotheses were tested:

$\mathrm{H}_{1}-\mathrm{CMD}$ prevalence rates from extended families are lower than those from nuclear families, and the rates from nuclear families are lower than those from partial families.

$\mathrm{H}_{2}$ - Children in smaller families exhibit a higher prevalence of CMD than children in larger families.

$\mathrm{H}_{3}$ - Prevalence of neurotic and psychosomatic disorders is lower for children in larger families than for those in smaller families.

\section{Method}

\section{The research setting}

A ... is an urban neighborhood of Salvador, capital city of the state of Bahia, Brazil. The population of the area, at the time of data collection, was about 60,000 with $47 \%$ of the population under 15 years of age. The average monthly income of half of the population was below the regional minimum wage (about US \$ 900 annually), and only $58 \%$ of the adults were regularly employed. Only $11.3 \%$ of the adult population had completed elementary school. The overall illiteracy rate was $30 \%$. There is no sewage system or garbage collection in the area, and 37\% of the households have running water and $76 \%$ have electricity (Pugliese et al. 1969; Santana 1978).

\section{Sampling procedure}

The family group was the sampling unit and the sample universe was defined as all children between 5 and 14 years old residing in A .... Due to the deficient address system, a random surface sample, based on a detailed and recent map of the area was used. The map was divided into 936 equal surface subareas with a mean of 23 households in each subarea. Using random numbers, 95 subareas were selected and all children in each subarea were administered the screening instrument. The study sample size had been estimated at 800 subjects, $5 \%$ of a total population of about 16000 children, based on an expected point prevalence of child mental disorders of $10 \%$.

\section{The screening instrument}

For the screening phase, the QMPI (Questionário de Morbidade Psiquiátrica Infantil) was used. The QMPI is the only instrument of its kind in Portuguese which has been shown to be valid and reliable (Almeida-Filho 1981). The instrument was initially based on the tri-axial classification of child mental disorders proposed by a WHO research team (Rutter et al. 1969). The QMPI was developed through a pre- 
test and a field test in the same area of $\mathrm{A} . .$. , and its final form included 35 items, covering the following diagnoses: developmental disorders, behavior disorders, neurotic and psychosomatic disorders, organic brain disorders and mental subnormality. During a pretest with children under treatment and a control group, the instrument version selected for the fieldwork showed high sensitivity (97\%), high specificity $(77 \%)$ and a low overall misclassification rate (13.2\%). During the field test, the QMPI was administered to 103 children who, according to their scores, were then classified into two groups: suspected of CMD and nonsuspected. Child psychiatrists blindly examined all suspected and a random sample of nonsuspected subjects, providing a clinical diagnosis to revalidate that version of the QMPI. In this analysis, the instrument presented equivalent validity measures compared to its performance during the pretest (sensitivity: $92.2 \%$, specificity: $80 \%$, misclassification rate: $12.8 \%$ ). The reliability study revealed that the QMPI has a high test-retest reliability $(r=+0.78$ to +0.82$)$ and also a high interrater reliability for recognition of abnormality and level of severity $(\mathrm{Kw}=+0.74$ and $\mathrm{Kw}=+0.60$, respectively).

\section{The survey}

The survey was completed in two phases:

1. First phase - Trained interviewers (medical students in Psychiatry internships) administered a general questionnaire and the QMPI to all families in the sample in order to detect suspected cases of CMD. The mother was the informant of choice, but in her absence another adult in the household provided the information. In addition to family data, the general questionnaire included specific questions on demographic and socioeconomic variable as well as utilization of health services. Total scores were assigned to each child according to the QMPI administration guidelines (Almeida-Filho 1981), using specific weights for each item. The children were classified as suspected or nonsuspected in relation to a cut-off score of 11 . This cut-off point was assigned as the one which provided the highest sensitivity and specificity rates of the instrument in the pretest.

2. Second phase - All children considered as suspected cases and a subsample of nonsuspected children were given a psychiatric interview. Two child psychiatrists were used, each with more than 6 years of clinical psychiatric experience and more than 2 years of practice with children and adolescents in the area of Salvador. The author met with these psychiatrists for a series of training meetings to standardize their diagnostic criteria. Neither psychiatrist knew the QMPI score or classification status of any of the children, although they knew that some nonsuspected children were to be included. Also, the interviewers and their families were not informed of the scores, reinforcing the double-blind character of the psychiatric evaluation. The interview was nonstructured with a mean duration of $30 \mathrm{~min}$, and was completed in the presence of the child and one relative, generally the mother. In some cases an older sister, aunt, or grandmother (in one case the father) attended the interview.

\section{Data analysis}

Child mental disorders (CMD) were defined as the state or condition of mental disability diagnosed by a child psychiatrist after the subject was selected using the screening procedure (the QMPI). Mental disease was not viewed solely as a clinical entity, but rather as an epidemiological research category. In the following analysis, CMD is approached through two dependent variables: (a) level of severity, and (b) diagnosis. The severity of each case was rated on a 4-point scale by the psychiatrists. The criterion used was need for treatment. The following categories comprised the severity scale: (a) no apparent psychiatric condition $=0$; (b) dubious or trivial condition (not needing psychiatric care) $=1$; (c) moderate condition (in need of psychiatric care) $=3$; (d) severe condition (urgent or constant need for psychiatric care) $=4$. Diagnoses were the type of CMD recognized by the psychiatrist and defined according to the tri-axial classification of mental disorders in childhood (Rutter et al. 1969).

Due to the small relative frequency of developmental disorders, organic conditions and mental retardation, these categories were collapsed into "other disorders". Comparisons were made between other disorders and neurotic and psychosomatic disorders. The category "behavior disorders", although included in the tri-axial scheme, was not considered in this study because of the particular sociocultural characteristics of the population under study, where behavior disorders are generally undistinguishable from normal adaptive behavior due to poverty, discrimination and stigma.

Family size, with a range of 2 to 16 members, was considered an ordinal variable with three categories: (a) small family - familial group with less than 5 members; (b) medium family - familial group with 5 to 10 members; (c) large family - familial group consisting of more than 10 members.

Family type was classified into three categories, 
Table 1. Family size by child mental disorders (CMD)

\begin{tabular}{|c|c|c|c|c|c|c|c|c|}
\hline \multirow[t]{2}{*}{ CMD } & \multicolumn{6}{|c|}{ Family size } & \multirow{2}{*}{$\begin{array}{l}\text { Total } \\
(n=828)\end{array}$} & \\
\hline & Small & $(n=133)$ & Medium & $(n=484)$ & Large & $(n=211)$ & & \\
\hline & $n$ & $\%$ & $n$ & $\%$ & $n$ & $\%$ & $n$ & $\%$ \\
\hline $\begin{array}{l}\text { Severity }{ }^{\mathrm{a}} \text { : } \\
\text { Dubious/trivial }\end{array}$ & 29 & 21.8 & 68 & 14.0 & 13 & 6.2 & 110 & 13.3 \\
\hline Moderate/severe & 20 & 15.0 & 48 & 9.9 & 15 & 7.1 & 83 & 10.0 \\
\hline $\begin{array}{l}\text { Diagnosis }^{\mathrm{b}} \text { : } \\
\text { Neurotic and psychosomatic disorders } \\
\text { Other disorders }\end{array}$ & $\begin{array}{l}36 \\
13\end{array}$ & $\begin{array}{r}27.1 \\
9.7\end{array}$ & $\begin{array}{l}80 \\
36\end{array}$ & $\begin{array}{r}16.5 \\
7.4\end{array}$ & $\begin{array}{l}14 \\
14\end{array}$ & $\begin{array}{l}6.7 \\
6.6\end{array}$ & $\begin{array}{r}130 \\
63\end{array}$ & $\begin{array}{r}15.7 \\
7.6\end{array}$ \\
\hline All cases & 49 & 36.8 & 116 & 23.9 & 28 & 13.3 & 193 & 23.3 \\
\hline
\end{tabular}

Significance tests:

${ }^{\mathrm{a}} x^{2}=28.71 ;$ d.f. $=4 ; P<0.001$

${ }^{b} x^{2}=31.39 ;$ d.f. $=4 ; P<0.001$

Table 2. Family type by child mental disorders (CMD)

\begin{tabular}{|c|c|c|c|c|c|c|c|c|}
\hline \multirow[t]{2}{*}{ CMD } & \multicolumn{6}{|c|}{ Family type } & \multirow{2}{*}{\multicolumn{2}{|c|}{$\begin{array}{l}\text { Total } \\
(n=828)\end{array}$}} \\
\hline & Partial & $(n=61)$ & Nuclear & $(n=481)$ & Extended & $(n=286)$ & & \\
\hline & $n$ & $\%$ & $n$ & $\%$ & $n$ & $\%$ & $n$ & $\%$ \\
\hline $\begin{array}{l}\text { Severity }{ }^{\mathrm{a}} \text { : } \\
\text { Dubious/trivial }\end{array}$ & 8 & 13.1 & 70 & 14.6 & 32 & 11.2 & 110 & 13.3 \\
\hline Moderate/severe & 6 & 9.8 & 50 & 10.4 & 27 & 9.4 & 83 & 10.0 \\
\hline $\begin{array}{l}\text { Diagnosis }^{\mathrm{b}} \text { : } \\
\text { Neurotic and psychosomatic disorders } \\
\text { Other disorders }\end{array}$ & $\begin{array}{r}11 \\
3\end{array}$ & $\begin{array}{r}18.0 \\
4.9\end{array}$ & $\begin{array}{l}83 \\
37\end{array}$ & $\begin{array}{r}17.3 \\
7.7\end{array}$ & $\begin{array}{l}36 \\
23\end{array}$ & $\begin{array}{r}12.6 \\
8.0\end{array}$ & $\begin{array}{r}130 \\
63\end{array}$ & $\begin{array}{r}15.7 \\
7.6\end{array}$ \\
\hline All cases & 14 & 23.0 & 120 & 24.9 & 59 & 20.6 & 193 & 23.3 \\
\hline
\end{tabular}

Significance tests:

${ }^{\mathrm{a}} x^{2}=2.15 ;$ d.f. $=4 ; P>0.05$

${ }^{b} x^{2}=3.79 ; d . f=4 ; P>0.05$

and was also considered an ordinal variable: (a) partial family - familial group which has lost, by death or separation, one or both parents; (b) nuclear family - familial group consisting of father, mother and children; (c) extended family - familial group composed of parents and children, plus any other relative living in the household.

In these analyses, the age and sex of the child, as well as the social class of the family were treated as potential confounding variables. Social class was measured by the income-displacement (I-D) index. This index combines the average monthly per capita income (total family income divided by the number of family members) and the employment status of the major source of family income. Four levels of the I-D index were used:

I - Per capita income (FPCI) below the minimum wage, head of household $(\mathbf{H H})$ not regularly employed;

II - FPCI above minimum wage, $\mathrm{HH}$ not regularly employed;
III - FPCI below minimum wage, $\mathrm{HH}$ regularly employed;

IV - FPCI above minimum wage, $\mathrm{HH}$ regularly employed.

Indexes of socioeconomic status based on occupation and education employed in social research in industrialized countries are not appropriate for research in underdeveloped societies due to high illiteracy and unemployment rates.

Data analysis followed the general methodology of stratified analysis for control of extraneous variables with three ordinal levels of exposure for both family size and family type, and two levels for both diagnosis and severity of CMD. The overall measure of association of choice was the $\bar{M}$, which is equivalent to the average "regression" of $Y$ (the ordinal dependent variable) on $X$ (the independent variable), controlling for confounding variables. The corresponding measure of statistical significance for each $\bar{M}$ is a special case of the summary chi-square statistic (Kleinbaum and Kupper 1979). 
Table 3. Control table of family size and family type by level of severity:

\begin{tabular}{|c|c|c|c|c|c|c|c|}
\hline & \multicolumn{7}{|c|}{ Family size } \\
\hline \multirow{2}{*}{$\frac{\text { Family type }}{\text { Partial }}$} & \multicolumn{3}{|c|}{ Small } & \multicolumn{2}{|c|}{ Medium } & \multicolumn{2}{|c|}{ Large } \\
\hline & (a) & 9 & 37.5 & 3 & 13.0 & 2 & 12.5 \\
\hline & (b) & 4 & 16.7 & 1 & 4.3 & 1 & 6.2 \\
\hline & (c) & 24 & 100.0 & 23 & 100.0 & 16 & 100.0 \\
\hline \multirow[t]{3}{*}{ Nuclear } & & 27 & 34.6 & 82 & 24.3 & 11 & 17.5 \\
\hline & & 11 & 14.1 & 34 & 10.1 & 5 & 7.9 \\
\hline & & 78 & 100.0 & 338 & 100.0 & 63 & 100.0 \\
\hline \multirow[t]{3}{*}{ Extended } & & 13 & 41.9 & 31 & 25.2 & 15 & 11.4 \\
\hline & & 5 & 16.1 & 13 & 10.6 & 9 & 6.8 \\
\hline & & 31 & 100.0 & 123 & 100.0 & 132 & 100.0 \\
\hline
\end{tabular}

(a) All cases of child mental disorders

(b) Moderate and severe cases

(c) Cell total

Table 4. Control table of family size and family type by diagnosis

\begin{tabular}{|c|c|c|c|c|c|c|c|}
\hline \multirow{3}{*}{$\frac{\text { Family type }}{\text { Partial }}$} & \multicolumn{7}{|c|}{ Family size } \\
\hline & \multicolumn{3}{|c|}{ Small } & \multicolumn{2}{|c|}{ Medium } & \multicolumn{2}{|c|}{ Large } \\
\hline & (a) & 8 & 33.3 & 2 & 8.7 & 1 & 6.2 \\
\hline & (b) & 1 & 4.2 & 1 & 4.3 & 1 & 6.2 \\
\hline & (c) & 24 & 100.0 & 23 & 100.0 & 16 & 100.0 \\
\hline \multirow[t]{3}{*}{ Nuclear } & & 20 & 25.6 & 58 & 17.2 & 5 & 7.9 \\
\hline & & 6 & 7.7 & 25 & 7.4 & 6 & 9.5 \\
\hline & & 78 & 100.0 & 338 & 100.0 & 63 & 100.0 \\
\hline \multirow[t]{3}{*}{ Extended } & & 8 & 25.8 & 20 & 16.3 & 8 & 6.1 \\
\hline & & 6 & 19.3 & 10 & 8.1 & 7 & 5.3 \\
\hline & & 31 & 100.0 & 123 & 100.0 & 132 & 100.0 \\
\hline
\end{tabular}

(a) Neurotic and psychosomatic disorders

(b) All other diagnosis

(c) Cell total

\section{Results}

Crude rates of diagnostic category and level of severity by family size are presented in Table 1 . A significant association between family size and both dependent variables can be seen (with severity: $\chi 2=28.71, P<0.001$ - with diagnosis: $\chi 2=31.39$, $P<0.001)$. Larger families were associated with lower prevalence and severity of child psychiatric disorders. With regard to family type (see Table 2 ) children from nuclear families exhibited the highest prevalence of CMD (25.0\%) and those from extended families showed the lowest prevalence (21\%). Crude rates for neurotic and psychosomatic disorders were about equal for partial and nuclear families, while extended families were associated with a slightly decreased prevalence of about $13 \%$. The opposite trend is evidenced for all other diagnoses. Severity ratings for dubious and trivial categories were highest (14.6\%) in nuclear families and lowest
$(11.2 \%)$ for extended families. None of these differences reached levels of statistical significance.

Crude rates, however, can be influenced by confounding factors. The first potential confounding variables examined were the age and sex of the child. Family size was associated with age $(\chi 2=6.53, P<$ 0.05 ), but no significant relationship was found between level of severity and age of the child $(\chi 2=2.59$, $P>0.05$ ). There was also no significant relationship between sex with family size $(\chi 2=0.79, P>0.05)$ or between sex and level of severity $(\chi 2=6.14, P>$ $0.05)$. Family size and the I-D index were related $(\chi 2=48.64, P<0.001)$ but no significant association was found between level of severity and the socioeconomic index $(\chi 2=6.43, P>0.05)$.

Also, it is necessary to consider family type as a major potential source of confounding in the associations. As seen in Table 3 , when controlling for family type, rates for all cases of CMD show a marked decrease across family size categories $(\chi 2=15.56, P<0.001, \bar{M}=-0.19)$. A similar, though weaker trend was evident for moderate and severe cases $(\chi 2=4.14, P<0.05, \bar{M}=-0.14)$. Conversely, when controlling for family size, no apparent trend was still evident in prevalence related to family type. For all cases, no significant relationship was found $(\chi 2=0.19, P>0.40)$. The same was true for moderate and severe cases $(\chi 2=0.005, P>0.90)$.

Hypothesis 3 proposed that large, extended families would be associated with lower rates of neurotic and psychosomatic disorders. Table 4 shows diagnostic categories, controlling for family size and family type. When controlling for the potential confounding, a consistent decrease across family size may be seen for neurotic and psychosomatic disorders $(\chi 2=17.35, P<0.001, \bar{M}=-0.24)$. This negative relationship was particularly apparent for partial families, where the excess "risk" was approximately $27 \%$ greater than for extended families. No consistent trend was seen for any of the other diagnoses $(\chi 2=0.93, P>0.05)$. Controlling for family size, no significant association for either psychosomatic disorders or for other diagnoses was found $\left(\chi^{2}=0.40\right.$, $P>0.05$, and $\chi 2=0.93, P>0.05$, respectively). Neurotic and psychosomatic disorders showed a slight excess in small, partial families, while the opposite trend may be seen in medium-sized families. No trend was apparent for large families.

Table 4 also shows that only in extended families was a negative association between family size and other diagnoses apparent. Partial and nuclear families showed slight increases in other diagnoses across family size. The overall measure of association was positive $(\bar{M}=+0.16)$ and reached significance $(\chi 2=6.10, P<0.01)$. When controlling for family 
type, family size showed no significant association with $\operatorname{CMD}(\bar{M}=+0.07, \chi 2=0.61, P>0.05)$.

Returning briefly to the possible role of social class (I-D index) in influencing the family size and type, a positive, but nonsignificant relationship was found between family type and all severity levels when controlling for social class $(\chi 2=2.53, P>0.05$, $\bar{M}=+0.17$ ). When the association between family size and severity was controlled for social class, however, a significant, negative association $(\chi 2=28.23$, $P<0.0005, \bar{M}=-0.27$ ) was still found. For moderate and severe cases examined separately, the same, though less strong relationship prevailed $(\chi 2=5.28$, $P<0.025, \bar{M}=-0.17)$. Controlling for social class, a strong negative association was also found between family size and neurotic and psychosomatic disor$\operatorname{ders}(\chi 2=27.14, P<0.0005, \bar{M}=-0.31)$.

\section{Discussion}

Crude prevalence rates supported both hypotheses 2 and 3. Partial support was also found for hypothesis 1 due to some indications that the extended family was more protective with regard to CMD than other family types. However, nuclear families did not show lower prevalence rates than partial families, as expected. The potential confounding effects of demographic and socioeconomic factors was assessed by utilizing controlling procedures throughout the analysis.

Age was not significantly related to severity of CMD, and sex showed no significant relationship to either family size or severity, thus allowing age and sex to be discarded as confounders. Family type and social class, however, were found to be potentially affecting the association between family size and CMD, and had to be considered further. Throughout the analysis, the findings still provided support for hypothesis 2 only. Even when controlling for both potential confounders, neither the trend nor the significance levels of the hypothesized relationship changed. The lack of association between family type and CMD variables (hypothesis 1) could have been the result of the confounding effect of social class. However, after controlling for the influence of the I-D index, the relationship remained nonsignificant.

The income-exclusion index (I-E) indeed shows a more specific pattern. While there is no significant relationship between the I-E index and outcome variables of CMD, there is a strong, significant association of the I-E index with both family size and family type. Social class, as measured by the I-E index, may influence the observed prevalence rates by acting through family size, family type, or through both variables. Nevertheless, a degree of caution must be exercised in interpreting the present results. With this in mind, a more detailed examination of the relationship among independent variables and severity of CMD is indicated.

The first outcome variable considered is level of severity of CMD. Examining the effect of family size on level of severity and controlling for the effect of family type, a strong and significant association was found for both categories: (a) all disorders, and (b) moderate and severe cases. Conversely, no significant relationship was found when controlling for family size and examining the effect of family type on level of severity. Thus, hypothesis 1 is not supported by the prevalence data for severity ratings of CMD; hypothesis 2 is strongly supported when controlling for the joint effects of both independent variables.

Hypothesis 3 suggested that large, extended families would be associated with lower rates of neurotic and psychosomatic disorders.

Analysis of neurotic and psychosomatic disorders provided some evidence in this respect. When controlled for family type, the trend suggested in hypothesis 2 (association between family size and CMD) was even stronger for this specific diagnostic category than for the other. While controlling for family size, the family type effect did not reach statistical significance. Therefore, also for neurotic and psychosomatic disorders, only family size was of some importance in the association under study.

With regard to the effect of potential confounders, when controlling for social class, in moderate and severe cases only, family size revealed the same negative trend but at a lower level of significance $(P<0.025)$. Neurotic and psychosomatic disorders showed a consistent, negative trend for the effect of family size controlling for social class. Family type showed no influence upon CMD after social class was controlled.

The conclusion that social class was not a confounding factor in the present analysis was reached because, after controlling for its potential effect, the trends observed in the crude analysis remained. This does not imply that no relationship could exist between social class and CMD. Family size and family type are possible effect-modifiers to be considered in any research on socioeconomic factors and mental disorders. Social class was negatively correlated with both family size and family type. Using family income as the social class indicator and taking family size as a discrete variable (total number of members living in the household), the Pearson correlation was $r=-0.254(P<0.01)$. If the hypotheses of this study 
are correct, one may consider a protective effect of larger family size on the prevalence of CMD. The indirect path with two negative associations (social class $\rightarrow$ family size $\rightarrow$ CMD) may be confounding the direct relationship between social class and CMD, by masking or weakening a true negative association.

Some methodological problems should be considered. First, these data were cross-sectional, and the effect of selective migration might have influenced the composition of the sample. Second, these data come from a population with a cultural and socioeconomic background which is complexly related to the entire set of variables analyzed. The protective effect of family size on CMD demonstrated in these data, may not apply equally to children living in a highly developed country or in a completely different cultural setting. Third, as with most research on social factors and mental disorders, the results could have been confounded by other variables not controlled for in the analysis. Fourth, as far as the dependent variable is concerned, the fact that socioeconomic characteristics of the sample did not allow the inclusion of "personality or behavior disorders" might also have biased the results. Personality patterns related to this group of disorders may be more frequent in larger families, which appear beneficial in childhood, but in the long run they may turn out to be considerably pathological.

Comparison of the present study with the previous literature was somewhat difficult due to methodological differences. For instance, previous researchers presented several distinct ways of categorizing family size. As a result, the large family has been defined as composed of five or more children (Nye 1952), six or more children (Landis 1954), more than seven children (Ellis and Beechley 1951), or the variable has not been categorized at all (Suepsaman 1973; Hendricks 1977). In addition, the age range of the sample and the definition of case have differed markedly among those studies.

Regarding results, four studies have supported the hypothesis of positive association between family size and CMD (Hendricks 1977). A closer examination of their designs reveals that quite different outcome variables were utilized. Hawkes et al. (1958) used the Rogers Test of Personality Adjustment to study "favorable relations with parents and siblings." Nye (1952) investigated normal adjustment of adolescents to parents with a nonvalidated 67 -item questionnaire. Spence et al. (1954) examined, among other issues, the relationship of family size to maternal care, and not to poor child mental health. Only Suepsaman (1973) investigated a comparable question through a cross-sectional study carried out in
Bangkok, Thailand, which lent support to the hypothesis of a detrimental effect of family size on CMD. In that survey, the CHOS (Children's Health Opinion Survey) was used to divide the sample into high-stress and low-stress groups without further clinical validation of the classification scheme.

Clinical research such as that of Ellis and Beechley (1951), Bossard and Boll (1956), and Tuckman and Regan (1967) tends to be in agreement with the findings of this study. Ellis and Beechley (1951), for instance, carried out a case-register study of 1000 children, using a family size categorization comparable to that of the present study. After controlling for age, sex, and intelligence, a significant negative association between family size and children's "emotional disturbance" was found. Similarly, Tuckman and Regan (1967) concluded that rates of anxiety and neurotic symptoms decreased with increasing family size. These findings, however, might have been biased by the nature of the sampleschildren referred to outpatient psychiatric clinics.

In addition to Suepsaman's (1973) study mentioned above, two other researchers have employed the CHOS. Leighton (1972), without testing for statistical significance, reported an association between high CHOS scores and both small and large families. Hendricks (1977), using multiple regression analysis on a small sample of black children, found a negative but nonsignificant correlation between family size and CHOS scores. The other studies reviewed did not present any consistent pattern relevant to family size and child mental health, and the methodological differences among them were also strikingly large. Two of the studies used "delinquent behavior" as the dependent variable (Nye 1958; Templeton 1962), while another studied teenagers' social adjustment to adulthood (Landis 1954), and another assessed the effect of family size on parents' acceptance of children in a Puerto Rican sample (Nuttall et al. 1971).

The present study seems to be the first on family variables and mental health which utilized psychiatric interviews to assess the children's psychological status. The double-blind interview procedures employed in this study could have reduced the biases implicit in the classical psychiatric diagnosis. The study design also seems to have been successful in testing specific hypotheses on prevalence data, thus combining descriptive and analytical strategies of epidemiological research.

In conclusion, the association of prevalence and severity of CMD with family size found in this study, at least for some specific psychopathological conditions, may reflect the greater availability of social support in larger families. Further research which di- 
rectly measures family social support is needed to clarify the relationship of support factors to family functioning and child mental health.

Alternative explanations, however, should be considered in interpreting these results. Larger families could be, in fact, more stressful, thereby leading to a higher degree of adaptation of their children. Also, the levels of family stress could be better correlated with other family variables, such as adult/child ratio, instead of overall family size. Thus, further research which approaches family stress through indicators more precise than the ones employed in this study are recommended. In addition, other questions must be considered in further investigations. What is the role of marital stability in affecting child mental health outcome and family size? Could the occurrence of problem children reduce further fertility? How does the birth order of a problem child influence family size?

Family composition and size, as well as other family variables, are a function of the group cultural and economic background. This does not mean that the A ... sample was a unique and isolated population group and that the results from this study cannot be generalized to other areas. Because of the increasing massive urbanization, similar settlements can now be found on the periphery of most major cities of Third World countries. The findings of this study using the A ... population may thus have broader implications for research and planning related to mental health problems in developing countries.

Acknowledgements. I am much indebted to Dr. Luiz Umberto Pinheiro and Vilma Santana, for their help in the development of the data collection. Charles K. Burnett and Janice Wheelon, from the University of North Carolina, co-authored an earlier version of this paper, and are mostly responsible for its translation. I would also like to thank one of the anonymous SP reviewers who gave many helpful suggestions for improving the manuscript.

\section{References}

Almeida Filho N de (1981) Development and assessment of the QMPI: Brazilian children's behavior questionnaire for completion by parents. Soc Psychiatry 16: 205-210

Baldassare M (1979) Residential crowding in urban America. University of California Press, Berkeley

Bossard JHS, Boll ES (1956) The large family system. University of Pennsylvania Press, Philadelphia

Cassel J (1971) Health consequences of population density and crowding. In: National Academy of Sciences. Rapid population growth: consequences and policy applications. Johns Hopkins Press, Baltimore

Cassel J (1973) The relationship of the urban environment to health: implications for prevention. Mt Sinai J Med 40: $539-450$

Cassel J (1976) The contribution of the social environment to host resistance. Am J Epidemiol 104: 107-123

Chombart de Lauwe P (1960) Famille et habitation. Editions du CNRS, Paris
Christensen HT (1968) Children in the family: relationship of number and spacing to marital success. J Marriage Family 30: $283-280$

Ellis A, Beechley RM (1951) A comparison of child guidance clinic patients coming from large, medium, and small families. $\mathbf{J}$ Gen Psychol 79: 131-144

Hassan R (1977) Social and psychological implications of high population density. Civilisations $28: 228-242$

Hawkes GR, Burchinal L, Gardner B (1958) Size of family and adjustment of children. Marriage Family Living 20: 65-68

Hendricks LE (1977) The effects of family size, child spacing and family density on stress in low income black mothers and their preadolescent children. Unpublished Doctoral Dissertation, University of North Carolina, Chapel Hill

Kaplan E, Cassel J, Gore S (1977) Social support and health. Med Care 15: 47-58

Kleinbaum D, Kupper L (1979) Advanced methods in epidemiology (EPI 268). University of North Carolina School of Public Health, Chapel Hill, NC (Mimeo)

Landis PH (1954) Teenage adjustments in large and small families. Washington State University Agricultural Experiment Stations. Bulletin No. 549

Leighton DC (1972) Measuring stress levels in school children as a program-monitoring device. Am J Publ Health 62: 799-804

Nuttall RL, Nuttall EV, Sweet PR (1971) Correlates of family size and expected family size among Puerto Rican youth. The Institute of Human Sciences at Boston College, Chestnut Hill, MA

Nye FI (1952) Sibling number, broken homes and adjustment. Marriage Family Living 14: 327-330

Nye FI (1958) Family relationships and delinquent behavior. Wiley and Sons, New York.

Pugliese C (1969) Plano de desenvolvimento comunitario do nordeste de Amaralina. Anals, Seminario ABEM, Sao Paulo

Rutter M (1972) Maternal deprivation revisited. Penguin Press, Baltimore

Rutter M, Lebovici S, Eisenberg L, Snezneuskij A, Sadoun R, Brooke E, Lin T (1969) A tri-axial classification of mental disorders in childhood: an international study. J Child Psychol Psychiatry 10: 41-61

Santana V (1978) Estudo de epidemiologia das doencas mentais em um bairro de Salvador: Nordeste de Amaralina. Unpublished Master's Thesis, University of Bahia

Schmitt RC (1966) Density, health and social disorganization. J Am Inst Planners 20: 273-283

Spence JC, Walton WS, Miller FJW, Court SDM (1954) A thousand families in Newcastle-Upon-Tyne. Oxford University Press, London

Suepsaman B (1973) The study of stress in Thai children: an epidemiological study of Thai children in Bankok, Thailand. Unpublished Doctoral Dissertation, University of North Carolina, Chapel Hill

Templeton JA (1962) Influence of family size on some aspects of teen-agers' attitudes, behavior and perceptions of home life. Family Life Coordinator 3: 51-57

Touliatos J, Lindholm BW (1980) Birth order, family size, and children's mental health. Psychol Reports 46: 1097-1098

Tuckman J, Regan RA (1967) Size of family and behavior problems in children. J Gen Psychol 111: 151-160

Accepted 17 May 1983

Naomar de Almeida-Filho

Rua Padre Feijó, $29-4^{\circ}$. andar - Anexo II

Faculdade de Medicina da UFBA

Canela, Salvador, Bahia

Brazil 40000 